18,12

\title{
Термофорез одноатомных частиц в открытых нанотрубках
}

\author{
() А.В. Савин ${ }^{1,2}$, О.И. Савина ${ }^{2}$ \\ ${ }^{1}$ Федеральный исследовательский центр химической физики им. Н.Н. Семенова РАН, \\ Москва, Россия \\ ${ }^{2}$ Российский экономический университет им. Г.В. Плеханова, \\ Москва, Россия \\ E-mail: asavin@center.chph.ras.ru
}

Поступила в Редакцию 16 ноября 2020 г.

В окончательной редакции 16 ноября 2020 г.

Принята к публикации 20 ноября 2020 г.

\begin{abstract}
Методом молекулярной динамики показано, что термофорез частиц (атомов) внутри одностенных углеродных нанотрубок (УНТ) обладает высокой эффективностью. Помещение частицы внутрь УНТ, участвующей в теплопереносе, приводит к ее движению в направлении потока тепла с постоянной скоростью, величина которой слабо зависит от длины нанотрубки. Тепловой поток вдоль УНТ приводит к образованию для частиц внутри нее постоянной силы термофореза, направление которой совпадает с направлением теплопереноса. Одноатомность частицы позволила численно вычислить эту силу и определить вклад в нее взаимодействия с каждым тепловым фононом нанотрубки. Показано, что величина силы практически полностью определяется взаимодействием частицы с длинноволновыми изгибными фононами нанотрубки, обладающими большой длиной пробега. Поэтому скорость движения частицы и величина силы термофореза слабо зависят от длины нанотрубки, а определяются разницей температур на ее концах. В силу этого режим термофореза частиц внутри нанотрубок является баллистическим, а не диффузионным.
\end{abstract}

Ключевые слова: нанотрубки, термофорез, теплоперенос, фононы нанотрубок.

DOI: 10.21883/FTT.2021.04.50726.239

\section{1. Введение}

Термофорезом называется направленное движение частиц, вызванное наличием внешнего теплового градиента. В настоящее время термофорез стал новым методом манипулирования наноразмерными частицами. Материалы с хорошей теплопроводностью и низким поверхностным трением, такие как нанотрубки, наноленты, листы графена хорошо подходят для использования их в качестве платформы для транспортировки тел наноразмера [1]. В настоящей работе мы рассмотрим термофорез частиц внутри углеродных одностенных нанотрубок - направленное движение частиц, вызванное наличием теплопереноса вдоль нанотрубок.

Углеродные нанотрубки (УНТ) представляют собой цилиндрические макромолекулы диаметром от половины нанометра и длиной до нескольких сантиметров. Подобные структуры были получены более 60 лет назад при термическом разложении окиси углерода на железном контакте [2]. Но сами нанотрубки получены только 29 лет назад как побочные продукты синтеза фуллерена $\mathrm{C}_{60}$ [3]. В настоящее время УНТ привлекают внимание благодаря своим уникальным свойствам [4], например, аномально высокой теплопроводности [5].

Тепловой поток вдоль нанотрубок может вызывать движение внутри нанотрубок наночастиц золота [6,7], нанокапель воды [8-13], вложенных нанотрубок [14-16] и молекул фуллерена $\mathrm{C}_{60}[17,18]$. Движение частиц внутри нанотрубок вызывается их взаимодействием с тепловыми фононами и происходит против градиента температуры: от теплого конца нанотрубки к холодному.

Термофорез наночастиц также может происходить вдоль нанолент графена. Здесь важно, чтобы был невозможен термически активированный отрыв частицы от наноленты, для этого необходимо достаточно сильное взаимодействие между ними. Вдоль нанолент может происходить направленный транспорт молекул фуллерена $[19,20]$, коротких нанотрубок [19], чешуек графена [21] и нанокластеров золота [22]. Моделирование показало, что движение наночастиц в первую очередь обеспечивается мягкими изгибными колебаниями наноленты [22].

Целью настоящей работы является численное моделирование термофореза одноатомной частицы (атома золота) внутри одностенных нанотрубок. Будет показано, что помещение частицы внутрь УНТ, участвующей в теплопереносе, приводит к ее движению с постоянной скоростью $v_{s}$ в направлении теплопереноса. Тепловой поток вдоль УНТ приводит к образованию для частиц внутри нее постоянной силы $F_{t h}$ (силы термофореза), направление которой совпадает с направлением теплопереноса. Одноатомность частицы позволит нам напрямую вычислить эту силу и определить вклад в нее взаимодействия с каждым тепловым фононом нанотрубки. Будет показано, что величина силы термофореза практически полностью определяется взаимодействием частицы с длинноволновыми изгибными фононами нанотрубки, обладающими большой длиной пробега. Поэтому скорость 
движения частицы $v_{s}$ и величина силы термофореза $F_{t h}$ слабо зависят от длины нанотрубки, а определяются разницей температур на ее концах.

\section{2. Модель}

Для моделирования динамики УНТ воспользуемся силовым полем, в котором разными потенциалами описываются деформации валентных связей и углов, торсионных и двугранных углов [23]. В этой модели энергия деформации валентных связей $\mathrm{C}-\mathrm{C}$ описывается потенциалом

$$
U_{1}\left(\mathbf{u}_{1}, \mathbf{u}_{2}\right)=\varepsilon_{1}\left\{\exp \left[-\alpha_{0}\left(\rho-\rho_{0}\right)\right]-1\right\}^{2},
$$

где трехмерные вектора $\mathbf{u}_{1}, \mathbf{u}_{2}$, задают координаты атомов углерода, образующих валентную связь, $\rho=\left|\mathbf{u}_{2}-\mathbf{u}_{1}\right|$ и $\varepsilon_{1}=4.9632 \mathrm{eV}$ - длина и энергия связи, $\alpha_{0}=1.7889 \AA^{-1}-$ параметр, определяющий жесткость связи, $\rho_{0}=1.418 \AA-$ равновесная длина связи.

Энергия деформации валентных углов $\mathrm{C}-\mathrm{C}-\mathrm{C}$ описывается потенциалом

$$
U_{2}\left(\mathbf{u}_{1}, \mathbf{u}_{2}, \mathbf{u}_{3}\right)=\varepsilon_{2}\left(\cos \varphi-\cos \varphi_{0}\right),
$$

где косинус валентного угла $\cos \varphi=-\left(\mathbf{v}_{1}, \mathbf{v}_{2}\right) /\left|\mathbf{v}_{1}\right|\left|\mathbf{v}_{2}\right|$, вектора $\mathbf{v}_{1}=\mathbf{u}_{2}-\mathbf{u}_{1}, \mathbf{v}_{2}=\mathbf{u}_{3}-\mathbf{u}_{2}$, а вектора $\mathbf{u}_{1}, \mathbf{u}_{2}, \mathbf{u}_{3}$ задают координаты атомов, образующих валентный угол, энергия $\varepsilon_{2}=1.3143 \mathrm{eV}$, равновесное значение угла $\varphi_{0}=120^{\circ}$.

Деформации торсионных и двугранных углов описываются потенциалом

$$
U_{3}\left(\mathbf{u}_{1}, \mathbf{u}_{2}, \mathbf{u}_{3}, \mathbf{u}_{4}\right)=\varepsilon_{3}(1-z \cos \phi),
$$

где $\quad \cos \phi=\left(\mathbf{w}_{1}, \mathbf{w}_{2}\right) /\left|\mathbf{w}_{1}\right|\left|\mathbf{w}_{2}\right|, \quad$ вектора $\quad \mathbf{w}_{1}=$ $=\left(\mathbf{u}_{2}-\mathbf{u}_{1}\right) \times\left(\mathbf{u}_{3}-\mathbf{u}_{2}\right), \quad \mathbf{w}_{2}=\left(\mathbf{u}_{3}-\mathbf{u}_{2}\right) \times\left(\mathbf{u}_{3}-\mathbf{u}_{4}\right)$, множитель $z=1$ для двугранного угла (равновесное значение угла $\left.\phi_{0}=0\right)$ и $z=-1$ для торсионного угла $\left(\phi_{0}=\pi\right)$, энергия $\varepsilon_{3}=0.499 \mathrm{eV}$ (трехмерные вектора $\mathbf{u}_{1}, \ldots, \mathbf{u}_{4}$ задают координаты атомов, образующих угол). Более детальное описание энергии деформации торсионных и двугранных углов дано в [23].

Невалентное взаимодействие атома золота с атомами нанотрубки опишем потенциалом Леннарда-Джонса

$$
V(r)=\varepsilon_{0}\left\{\left[\left(r_{0} / r\right)^{6}-1\right]^{2}-1\right\},
$$

где $r$ - расстояние между взаимодействующими атомами, энергия взаимодействия $\varepsilon_{0}=0.01273 \mathrm{eV}$, равновесное расстояние $r_{0}=3.3610 \AA$ [24].

Для определенности рассмотрим динамику атома $\mathrm{Au}$ внутри открытой одностенной углеродной нанотрубки с индексом хиральности $(m, m)$ (внутри УНТ со структурой „кресло“). Вид системы $\mathrm{Au}+$ УНТ $(5,5)$ представлена на рис. 1.

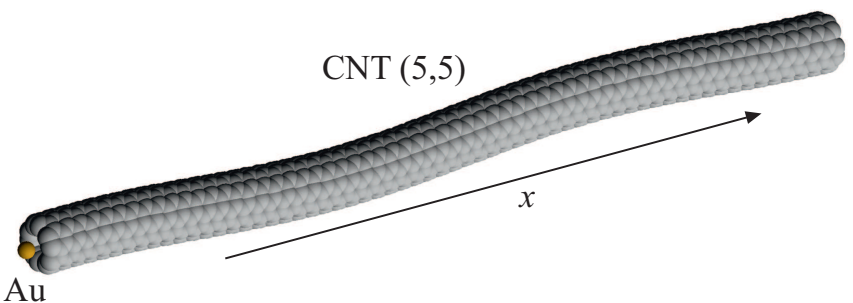

Рис. 1. Атом золота Аи и открытая одностенная УНТ с индексом хиральности $(5,5)$. У нанотрубки показаны изгибные колебания, ответственные за термофорез частиц внутри нее.

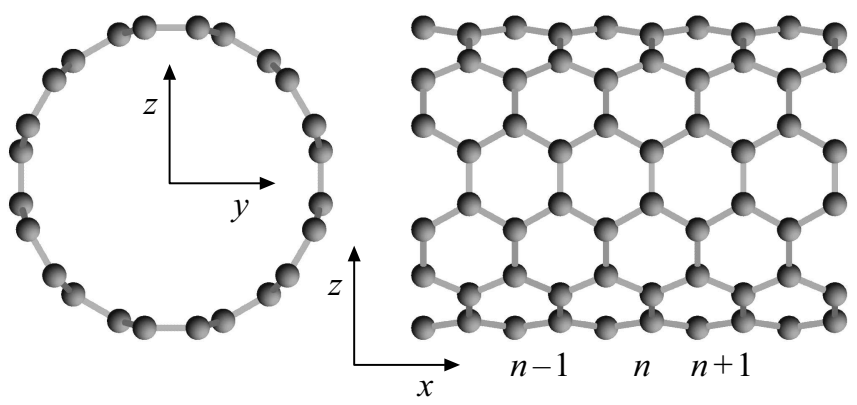

Рис. 2. Схематический вид одностенной углеродной нанотрубки с индексом хиральности $(6,6)$. Цилиндрическая структура нанотрубки образована продольным сдвигом на шаг $a=0.246 \mathrm{~nm}$ циклических зигзагообразных цепочек структуры „кресло“ из атомов углерода (диаметр цепи $D=0.805 \mathrm{~nm}$ ). Нанотрубка лежит вдоль оси $x$, индекс $n$ нумерует поперечные кольца атомов.

Рассмотрим одностенную УНТ с индексом хиральности $(m, m)$, направленную вдоль оси $x-$ см. рис. 2. Цилиндрическая структура нанотрубки образуется периодическим повторением вдоль оси $x$ поперечных циклических зигзагообразных цепочек из $K=4 m$ атомов углерода (поперечный диаметр нанотрубки $D \approx \rho_{0} / \sin (\pi / 3 m)$, продольный шаг $\left.a \approx \rho_{0} \sqrt{3}\right)$. Пусть индекс $n$ нумерует поперечные кольца атомов со структурой „кресло“, а индекс $k$ - атомы в кольцах, тогда положение атомов каждого кольца можно описать вектором размерности $3 \times K$

$$
\mathbf{x}_{n}=\left\{\mathbf{u}_{n, k}=\left(x_{n, k}, y_{n, k}, z_{n, k}\right)\right\}_{k=1}^{K}
$$

(индекс $n$ нумерует атомы углерода вдоль, индекс $k-$ поперек нанотрубки).

Гамильтониан нанотрубки можно записать в виде

$$
H=\sum_{n=-\infty}^{\infty} h_{n}=\sum_{n=-\infty}^{\infty}\left[\frac{1}{2} M\left(\dot{\mathbf{x}}_{n}, \dot{\mathbf{x}}_{n}\right)+P\left(\mathbf{x}_{n-1}, \mathbf{x}_{n}, \mathbf{x}_{n+1}\right)\right],
$$

где первый член в сумме описывает кинетическую энергию атомов $n$-ой ячейки $(M-$ масса атома углерода), второй член $P\left(\mathbf{x}_{n-1}, \mathbf{x}_{n}, \mathbf{x}_{n+1}\right)-$ энергию взаимодействия атомов $n$-ой элементарной ячейки между собой и с атомами соседних ячеек. 
В основном состоянии каждая элементарная ячейка нанотрубки получается из предыдущей сдвигом на продольный шаг нанотрубки $a: \mathbf{x}_{n}^{0}=\mathbf{x}^{0}+a n \mathbf{e}_{x}$, где вектор $\mathbf{e}_{x}=\{(1,0,0)\}_{k=1}^{K}$. Для нахождения основного состояния (для нахождения продольного шага $a$ и вектора координат $\mathbf{x}^{0}$ ) нужно решить задачу на минимум энергии основного состояния

$$
P\left(\mathbf{x}^{0}-a \mathbf{e}_{x}, \mathbf{x}^{0}, \mathbf{x}^{0}+a \mathbf{e}_{x}\right) \rightarrow \min : \mathbf{x}^{0}, a .
$$

Задача (6) решалась численно методом сопряженного градиента.

\section{3. Дисперсионные кривые}

Пусть в основном состоянии атомы нанотрубки имеют координаты $\mathbf{x}_{n}^{0}=\mathbf{x}^{0}+a n \mathbf{e}_{x}, n=0, \pm 1, \pm 2, \ldots$ Введем $3 K$-мерный вектор $\mathbf{v}_{n}=\mathbf{x}_{n}-\mathbf{x}_{n}^{0}$, описывающий смещения атомов $n$-ой ячейки из своего положения равновесия. Тогда гамильтониан нанотрубки будет иметь вид

$$
H=\sum_{n}\left[\frac{1}{2} M\left(\dot{\mathbf{v}}_{n}, \dot{\mathbf{v}}_{n}\right)+U\left(\mathbf{v}_{n-1}, \mathbf{v}_{n}, \mathbf{v}_{n+1}\right)\right],
$$

где энергия взаимодействия соседних звеньев $U\left(\mathbf{v}_{1}, \mathbf{v}_{2}, \mathbf{v}_{3}\right)=P\left(\mathbf{x}^{0}-a \mathbf{e}_{x}+\mathbf{v}_{1}, \mathbf{x}^{0}+\mathbf{v}_{2}, \mathbf{x}^{0}+a \mathbf{e}_{x}+\mathbf{v}_{3}\right)$.

Гамильтониану (7) соответствует система уравнений движения

$$
\begin{aligned}
-M \ddot{\mathbf{v}}_{n}= & \frac{\partial}{\partial \mathbf{v}_{n}} H=U_{\mathbf{v}_{1}}\left(\mathbf{v}_{n}, \mathbf{v}_{n+1}, \mathbf{v}_{n+2}\right) \\
& +U_{\mathbf{v}_{2}}\left(\mathbf{v}_{n-1}, \mathbf{v}_{n}, \mathbf{v}_{n+1}\right)+U_{\mathbf{v}_{3}}\left(\mathbf{v}_{n-2}, \mathbf{v}_{n-1}, \mathbf{v}_{n}\right),
\end{aligned}
$$

где вектор частных производных $U_{\mathbf{v}_{1}}=\partial U\left(\mathbf{v}_{1}, \mathbf{v}_{2}, \mathbf{v}_{3}\right) / \partial \mathbf{v}_{i}$, $i=1,2,3$. В линейном приближении система уравнений движений (8) принимает вид

$$
-M \ddot{\mathbf{v}}_{n}=\mathbf{B}_{1} \mathbf{v}_{n}+\mathbf{B}_{2} \mathbf{v}_{n+1}+\mathbf{B}_{2}^{*} \mathbf{v}_{n-1}+\mathbf{B}_{3} \mathbf{v}_{n+2}+\mathbf{B}_{3}^{*} \mathbf{v}_{n-2},
$$

где матричные коэффициенты $\mathbf{B}_{1}=U_{\mathbf{v}_{1} \mathbf{v}_{1}}+U_{\mathbf{v}_{2} \mathbf{v}_{2}}+U_{\mathbf{v}_{3} \mathbf{v}_{3}}$, $\mathbf{B}_{2}=U_{\mathbf{v}_{1} \mathbf{v}_{2}}+U_{\mathbf{v}_{2} \mathbf{v}_{3}}, \mathbf{B}_{3}=U_{\mathbf{v}_{1} \mathbf{v}_{3}}$, а матрицы частных производных

$$
U_{\mathbf{v}_{i} \mathbf{v}_{j}}=\frac{\partial^{2} U}{\partial \mathbf{v}_{i} \partial \mathbf{v}_{j}}(\mathbf{0}, \mathbf{0}, \mathbf{0}), \quad i, j=1,2,3 .
$$

Решение системы линейных уравнений (9) может быть получено в виде волны

$$
\mathbf{v}_{n}=A \mathbf{w} \exp (i q n-i \omega t),
$$

где $A-$ амплитуда, $\mathbf{w}-$ нормированный безразмерный вектор $(|\mathbf{w}|=1), q \in[0, \pi]$ - безразмерное волновое число, $\omega$ - частота колебания. Подставив выражение (10) в систему (9), мы получим задачу на собственные значения

$$
\omega^{2} M \mathbf{w}=\mathbf{C}(q) \mathbf{w},
$$

где эрмитова матрица $\mathbf{C}(q)=\mathbf{B}_{1}+\mathbf{B}_{2} e^{i q}+\mathbf{B}_{2}^{*} e^{-i q}$ $+\mathbf{B}_{3} e^{2 i q}+\mathbf{B}_{3}^{*} e^{-2 i q}$.

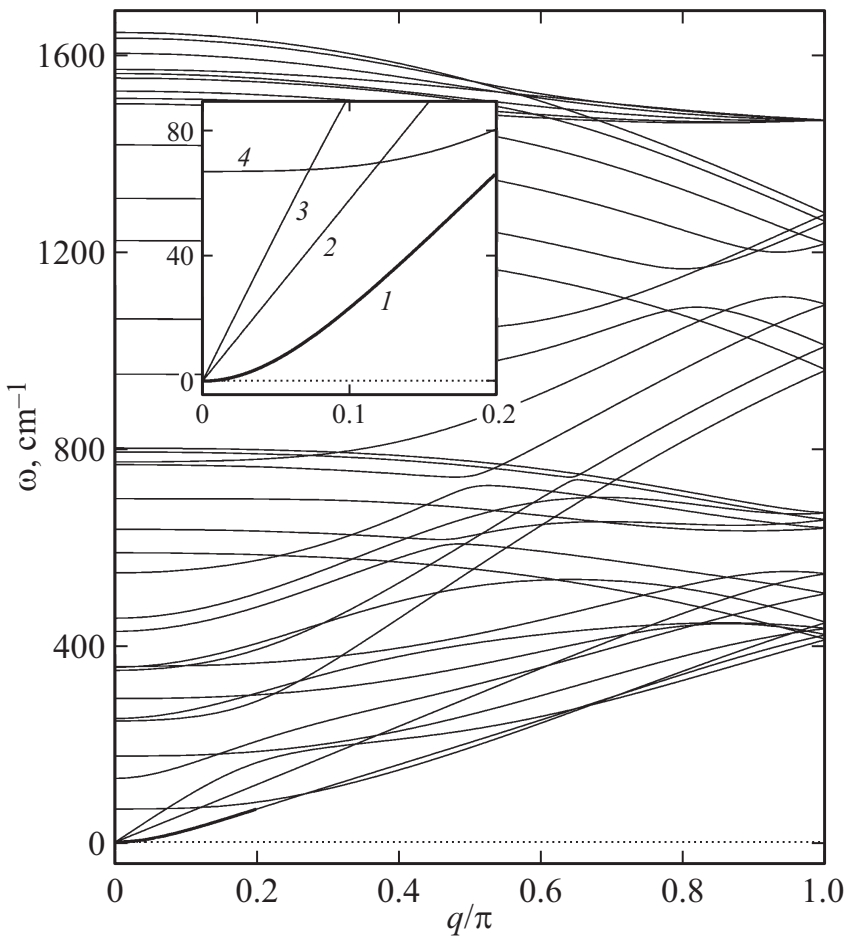

Рис. 3. Вид 60 дисперсионных кривых УНТ $(5,5)$. Двукратная кривая 1 соответствует изгибным, кривая 2 - крутильным акустическим, кривая 3 - продольным акустическим, кривая $4-$ оптическим радиальным колебаниям нанотрубки.

Решение задачи (11) позволяет найти все собственные частоты $\left\{\omega_{i}(q)\right\}_{i=1}^{3 K}$ и соответствующие собственные вектора $\left\{\mathbf{w}_{i}(q)\right\}_{k=1}^{3 K}$ для каждого значения волнового числа $q \in[0, \pi]$. Таким образом, для нахождения $3 K$ ветвей дисперсионной кривой мы должны при каждом фиксированном значении волнового числа $q$ численно найти все собственные значения эрмитовой матрицы $\mathbf{C}(q)$.

Вид дисперсионных кривых для нанотрубки $(5,5)$ представлен на рис. 3. Нанотрубка имеет частотный спектр $0 \leq \omega \leq 1642 \mathrm{~cm}^{-1}$. Из нулевой точки выходят: соответствующая изгибным (поперечным) колебаниям двукратная кривая $\omega_{1}(q)=\omega_{2}(q)$; кривая $\omega_{3}(q)$, соответствующая крутильным акустическим; кривая $\omega_{4}(q)$, соответствующая продольным акустическим колебаниям нанотрубки $\left(\omega_{1}(q)=\omega_{2}(q)<\omega_{3}(q)<\omega_{4}(q)\right.$ при $0<q<0.2)$. Скорости длинноволновых акустических колебаний $v_{3,4}=a \lim _{q \rightarrow 0} \omega_{3,4}(q) / q$, для крутильных колебаний $v_{3}=8770 \mathrm{~m} / \mathrm{s}$, для продольных $v_{4}=14277 \mathrm{~m} / \mathrm{s}$.

\section{4. Теплоперенос вдоль нанотрубок}

Гамильтониану нанотрубки (5) соответствует система уравнений движений

$$
-M \ddot{\mathbf{x}}_{n}=\frac{\partial}{\partial \mathbf{x}_{n}} H=\mathbf{F}_{n}=\mathbf{P}_{1, n+1}+\mathbf{P}_{2, n}+\mathbf{P}_{3, n-1},
$$

где функция $\quad \mathbf{P}_{i, n}=\mathbf{P}_{i}\left(\mathbf{x}_{n-1}, \mathbf{x}_{n}, \mathbf{n}_{n+1}\right), \quad \mathbf{P}_{i}=$ $=\partial \mathbf{P}\left(\mathbf{x}_{1}, \mathbf{x}_{2}, \mathbf{x}_{3}\right) / \partial \mathbf{x}_{i}, i=1,2,3$. 
Локальный тепловой поток через $n$-ую поперечную ячейку $j_{n}$ можно определить из уравнения изменения локальной плотности энергии $h_{n}$ :

$$
\frac{d}{d t} h_{n}=j_{n}-j_{n+1} .
$$

Из вида плотности энергии (5) и из системы уравнений движений (12) следует, что изменение энергии

$$
\begin{aligned}
\frac{d}{d t} h_{n} & =M \dot{\mathbf{x}}_{n} \ddot{\mathbf{x}}_{n}+\mathbf{P}_{1, n} \dot{\mathbf{x}}_{n-1}+\mathbf{P}_{2, n} \dot{\mathbf{x}}_{n}+\mathbf{P}_{3, n} \dot{\mathbf{x}}_{n+1} \\
& =-\mathbf{P}_{1, n+1} \dot{\mathbf{x}}_{n}-\mathbf{P}_{3, n-1} \dot{\mathbf{x}}_{n}+\mathbf{P}_{1, n} \dot{\mathbf{x}}_{n-1}+\mathbf{P}_{3, n} \dot{\mathbf{x}}_{n+1} .
\end{aligned}
$$

Из этого равенства и формулы (13) следует, что тепловой поток через $n$-ую поперечную ячейку

$$
j_{n}=\left(\mathbf{P}_{1, n}, \dot{\mathbf{x}}_{n-1}\right)-\left(\mathbf{P}_{3, n-1}, \dot{\mathbf{x}}_{n}\right) .
$$

Для прямого моделирования теплопереноса рассмотрим конечную нанотрубку длины $L=(N-0.5) a$ с фиксированными концевыми сегментами с индексами $n=1,2$ и $n=N-1, N$. Поместим первые $N_{t}=20$ сегментов в термостат Ланжевена температуры $T_{+}$, а последние - в термостат температуры $T_{-}$(разница температуры $\Delta T=T_{+}-T_{-} \geq 0$ ). Тогда для моделирования теплопереноса нужно будет численно проинтегрировать систему уравнений:

$$
\begin{aligned}
& M \ddot{\mathbf{x}}_{n}=-\mathbf{F}_{n}-\Gamma M \dot{\mathbf{x}}_{n}+\Xi_{n}^{+}, \quad 2<n \leq N_{t}, \\
& M \ddot{\mathbf{x}}_{n}=-\mathbf{F}_{n}, \quad N_{t}<n \leq N-N_{t}, \\
& M \ddot{\mathbf{x}}_{n}=-\mathbf{F}_{n}-\Gamma M \dot{\mathbf{x}}_{n}+\Xi_{n}^{-}, \quad N-N_{t}<n \leq N-2,
\end{aligned}
$$

где $\Gamma=1 / t_{r}-$ коэффициент трения (время релаксации $\left.t_{r}=0.4 \mathrm{ps}\right), \quad \Xi_{n}^{ \pm}=\left\{\xi_{n, k, i}^{ \pm}\right\}_{k=1, i=1}^{K, 3}-3 K$-мерный вектор нормально распределенных случайных сил, нормализованных условиями

$$
\left\langle\xi_{n, k, i}^{ \pm}\left(t_{1}\right) \xi_{m, l, j}^{ \pm}\left(t_{2}\right)\right\rangle=2 M \Gamma k_{B} T_{ \pm} \delta_{n m} \delta_{k l} \delta_{i j} \delta\left(t_{1}-t_{2}\right),
$$

( $k_{B}$ - постоянная Больцмана).

Возьмем начальные условия для системы уравнений движения (15), соответствующими основному состоянию нанотрубки $\left\{\mathbf{x}_{n}(0)=\mathbf{x}_{n}^{0}, \dot{\mathbf{x}}_{n}(0)=0\right\}_{n=1}^{N}$, и численно проинтегрируем систему до образования стационарного теплового потока. В центральной части наноленты $N_{t}<n \leq N-N_{t}$ произойдет образование температурного градиента и постоянного потока энергии. Распределение средних значений температуры и теплового потока вдоль нанотрубки находятся по формулам:

$$
\begin{gathered}
\bar{T}_{n}=\left\langle T_{n}\right\rangle=\lim _{t \rightarrow \infty} \frac{M}{3 K k_{B} t} \int_{0}^{t}\left|\dot{\mathbf{x}}_{n}(\tau)\right|^{2} d \tau, \\
\bar{J}_{n}=\left\langle J_{n}\right\rangle=\lim _{t \rightarrow \infty} \frac{a}{t} \int_{0}^{t} j_{n}(\tau) d \tau .
\end{gathered}
$$

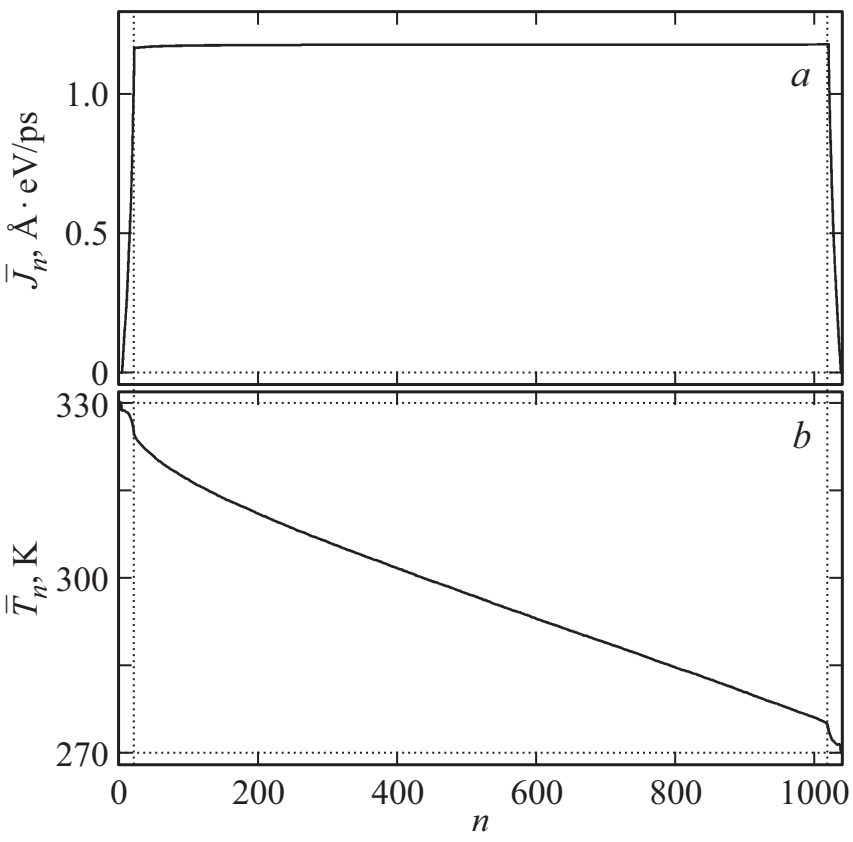

Рис. 4. Распределение вдоль УНТ $(5,5)$ локального теплового потока $\bar{J}_{N}(a)$ и локальной температуры $\bar{T}_{n}(b)$. Нанотрубка состоит из $N=1040$ поперечных ячеек (длина $L=(N-0.5) a=255.7 \mathrm{~nm}), \quad$ температуры термостатов $T_{ \pm}=300 \pm 30 \mathrm{~K}$, число краевых звеньев, взаимодействующих с термостатами $N_{t}=20$.

Распределение температуры и локального теплового потока вдоль нанотрубки показано на рис. 4. В центральной части нанотрубки тепловой поток не меняется: $\bar{J}_{n}=J$ при $N_{t}<n \leq N-N_{t}$. Независимость величины локального теплового потока $\bar{J}_{n}$ от номера ячейки $n$ может служить критерием образования стационарного теплового потока. Образующийся в центральной части нанотрубки градиент температуры может быть использован для нахождения коэффициента теплопроводности

$$
\kappa\left(N_{i}\right)=\left(N_{i}-1\right) J /\left(T_{N_{t}+1}-T_{N-N_{t}}\right) S,
$$

где $N_{t}=N-2 N_{t}-$ число поперечных ячеек в центральной части нанотрубки, $S=\pi\left(R+r_{C}\right)^{2}-$ площадь поперечного сечения нанотрубки $(R=D / 2-$ радиус нанотрубки, $r_{C}=1.85 \AA-$ радиус Ван-дер-Ваальса атома углерода).

Углеродные нанотрубки обладают аномально высокой теплопроводностью $[5,25]$. Прямое моделирование теплопереноса показывает [26], что коэффициент теплопроводности идеальной нанотрубки $\kappa(N)$ монотонно увеличивается с увеличением ее длины $L=(N-0.5) a$ как логарифм длины:

$$
\kappa(N) \sim \ln N \text { при } N \rightarrow \infty .
$$

При длинах $L<640 \mathrm{~nm}$ (при $N \leq 2600))$ теплопроводность растет явно медленнее любой степенной функции 


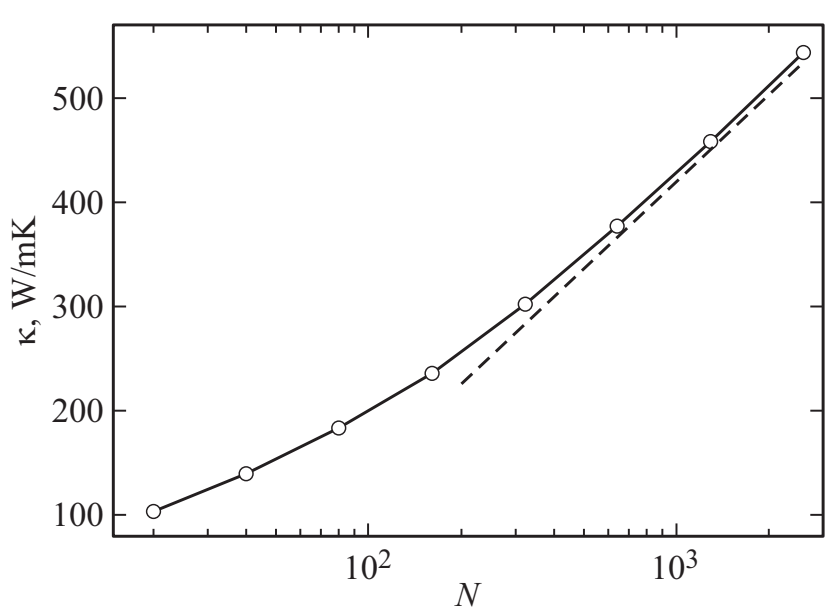

Рис. 5. Зависимость коэффициента теплопроводности УНТ $(6,6) \kappa$ от числа поперечных ячеек $N$ при температуре $T=300 \mathrm{~K}$. Штриховая линия дает зависимость $\kappa=121 \ln N$.

длины. На этих длинах рост теплопроводности хорошо аппроксимируется логарифмом ее длины - см. рис. 5.

\section{5. Движение одноатомной частицы внутри нанотрубки}

Рассмотрим движение частицы, состоящей из одного атома золота, внутри нанотрубки, по которой происходит теплоперенос. Для этого после образования в УНТ стационарного теплового потока поместим частицу в левый (более теплый) конец нанотрубки - см. рис. 1. Отметим, что в нанотрубке с индексом хираль-

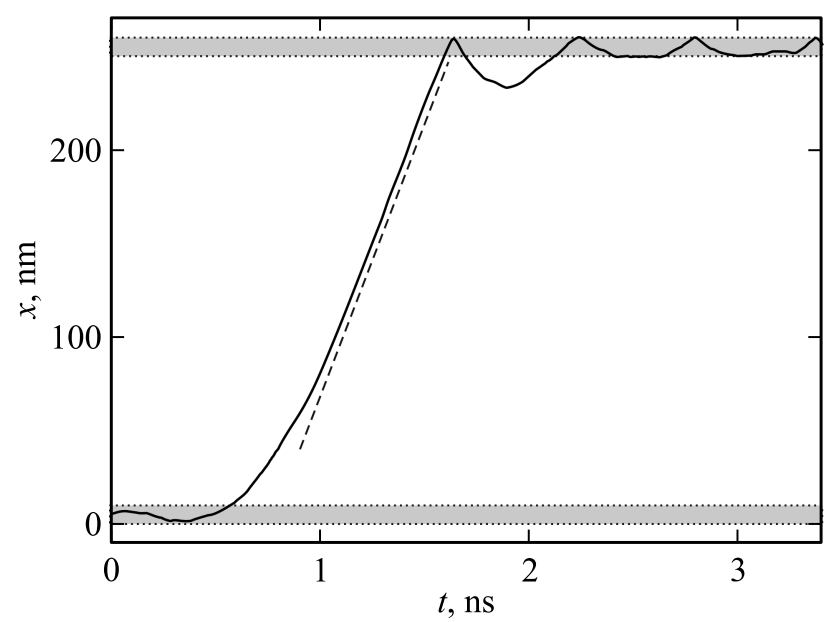

Рис. 6. Движение атома Аu внутри УНТ $(5,5)$ при длине нанотрубки $L=255.7 \mathrm{~nm}(N=1040)$ и температуре термостатов $T_{ \pm}=300 \pm 15 \mathrm{~K}$. Показана зависимость от времени $t$ координаты атома $x$. Серым цветом показаны концы нанотрубки, взаимодействующие с термостатами. Скорость установившегося движения атома $v_{s}=290 \mathrm{~m} / \mathrm{s}$ (штриховая линия дает зависимость $\left.v_{s} t\right)$. ности $(\mathrm{m}, \mathrm{m})$ размер внутренней полости достаточен для помещения в нее атома $\mathrm{Au}$ только при $m \geq 5$. Заметим, что если атом поместить на внешнюю поверхность нанотрубки, то тепловые колебания будут приводить к его отрыву. Так помещение атома $\mathrm{Au}$ на внешнюю поверхность нанотрубки $(5,5)$ приводит к энергетическому выигрышу $\Delta E=0.142 \mathrm{eV}$, сравнимому с энергией тепловых колебаний $3 k_{B} T=0.078 \mathrm{eV}$ при температу-

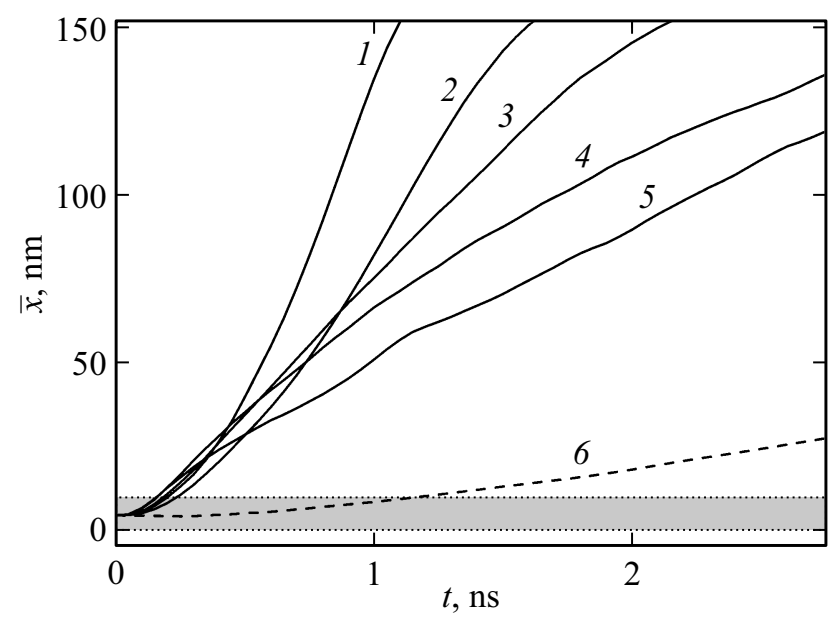

Рис. 7. Зависимость от времени $t$ усредненной координаты атома золота $\bar{x}$ при движении внутри УНТ $(m, m)$ при $m=5,6,7,8,12$ (кривые 1,2, 3, 4,5). Длина нанотрубки $L=255.7 \mathrm{~nm}\left(N=1040, N_{t}=20, T_{ \pm}=300 \pm 30 \mathrm{~K}\right)$. Серым цветом показан левый конец нанотрубки, взаимодействующий с теплым термостатом. Значения координаты $x$ усреднялись по 256 независимым реализациям термализации термостатов. Штриховая линия 6 дает зависимость для нанотрубки $(5,5)$ при учете только продольных движений ее атомов.

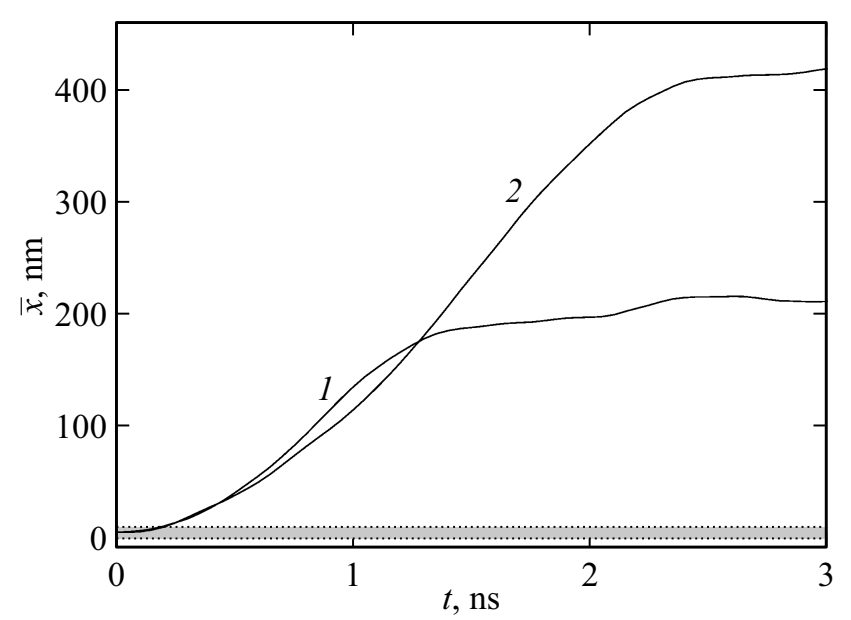

Рис. 8. Зависимость от времени $t$ усредненной координаты атома золота $\bar{x}$ при его движении внутри УНТ $(5,5)$ при ее длине $L=255.7$ и $501.7 \mathrm{~nm}(N=1040$ и 2040) - кривые 1 и 2. Температура термостатов $T_{ \pm}=300 \pm 30 \mathrm{~K}$. Серым цветом показан левый конец нанотрубки, взаимодействующий с теплым термостатом. Значения координаты $x$ усреднялись по 256 независимым реализациям термализации термостатов. 
pe $T=300 \mathrm{~K}$. Помещение атома внутрь нанотрубки приводит к энергетическому выигрышу $\Delta E=0.547 \mathrm{eV}$. Внутри нанотрубки отрыву частицы от ее поверхности также всегда препятствует ее замкнутая цилиндрическая форма.

Пример движения атома внутри нанотрубки, участвующей в теплопереносе, показан на рис. 6. Под действием теплового потока атом Аи начинает монотонно двигаться в направлении теплопереноса (от теплого края к холодному). Вначале скорость движения монотонно увеличивается, достигает максимального значения $v_{s}$ и далее остается постоянной. После достижении правого края нанотрубки атом начинает колебаться у края.

Увеличение диаметра нанотрубки $(m, m)$ (увеличение индекса $m$ ) приводит к уменьшению скорости движения атома - см. рис. 7. Средняя скорость установившегося движения $\bar{v}_{s}=216,136,86,71,66 \mathrm{~m} / \mathrm{s}$ при $m=5,6,7,9,12$. Уменьшение скорости связано с уменьшением энергии взаимодействия атома с нанотрубкой при увеличении ее радиуса. С другой стороны, увеличение длины нанотрубки практически не приводит к уменьшению скорости $v_{s}-$ см. рис. 8 .

\section{6. Сила термофореза}

Результаты моделирования позволяют заключить, что движение частицы внутри нанотрубки под действием теплового потока качественно может быть описано как ее движение в вязкой среде под действием постоянной силы:

$$
M_{0} \ddot{x}=F_{t h}-\gamma \dot{x},
$$

где $M_{0}$ - масса частицы (атома $\left.\mathrm{Au}\right), F_{t h}$ - эффективная сила термофореза, $\gamma-$ коэффициент трения, характеризующий интенсивность взаимодействия частицы с тепловыми колебаниями атомов нанотрубки. Из уравнения (17) следует, что скорость установившегося движения атома $v_{s}=F_{t h} / \gamma$.

Одноатомность частицы позволяет найти значение силы термофореза $F_{t h}$ численно. Для этого нужно частицу расположить внутри нанотрубки по ее центру (взять координату $x=(N-0.5) a / 2)$. Зафиксировать эту координату, но разрешить движение по осям $y$ и $z$. Далее промоделировать теплоперенос вдоль нанотрубки и найти среднее значение компоненты силы $F_{x}$, действующей на частицу со стороны атомов нанотрубки: $F_{t h}=\left\langle F_{x}\right\rangle$.

Из асимптотики (16) следует, что при фиксированных значениях температур термостатов тепловой поток вдоль нанотрубки с увеличением ее длины будет убывать как $J(N): \ln (N) / N$ при $N \rightarrow \infty$. Моделирование теплопереноса показывает, что сила термофореза $F_{t h}$ убывает значительно медленнее - см. рис. 9. Таким образом, сила термофореза непропорциональна величине теплового потока, а пропорциональна только разнице температур термостатов $F_{t h}: \Delta T$ при $\Delta T=\left(T_{+}-T_{-}\right) \rightarrow 0$.

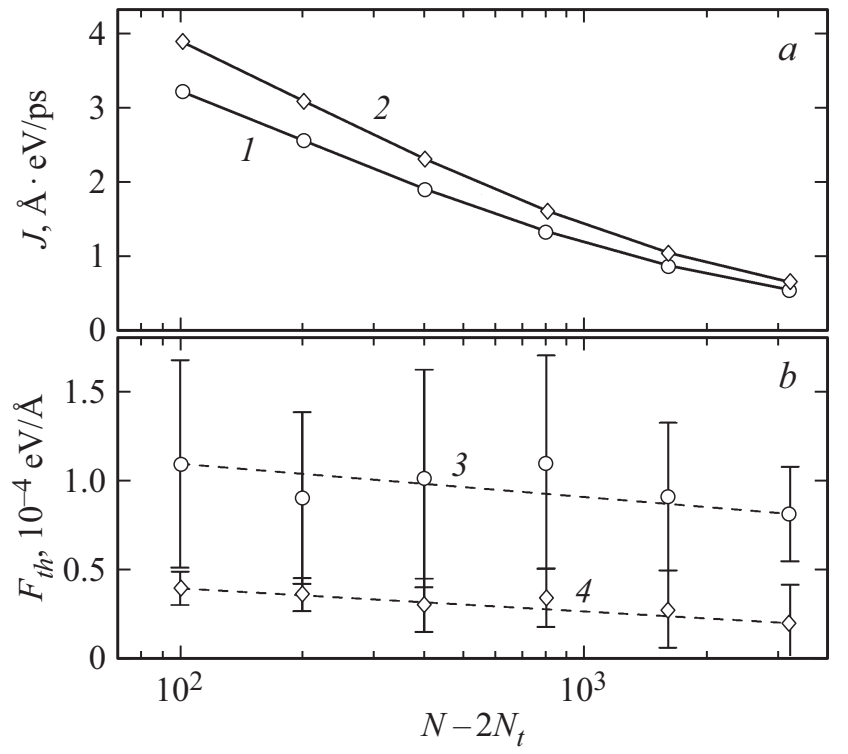

Рис. 9. Зависимость теплового потока $J(a)$ и силы термофореза $F_{t h}(b)$ от числа поперечных ячеек нанотрубки $N-2 N_{t}$ между ее концами, взаимодействующими с термостатами температуры $T_{ \pm}=300 \pm 30 \mathrm{~K}\left(N_{t}=20\right)$. Кривые 1 и 3 дают зависимости для нанотрубки $(5,5)$, кривые 2 и $4-$ для нанотрубки $(6,6)$. Вертикальные линии на части $(b)$ дают интервалы отклонений сил $F_{t h}$ от своих средних значений.

Рассходимость теплопроводности нанотрубки (медленное убывание $J(N)$ при $N \rightarrow \infty$ ) обусловлена баллистическим потоком длинноволновых акустических фононов. Более медленное убывание силы $F_{t h}$ при $N \rightarrow \infty$ говорит о том, что термофорез обусловлен взаимодействием частицы только с частью длинноволновых фононов. Для того чтобы понять, взаимодействие с какими фононами нанотрубки обеспечивает термофорез, оценим взаимодействие частицы (атома $\mathrm{Au})$ с каждым фононом.

Одноатомность частицы позволяет численно найти среднее значение силы ее взаимодействия с бегущей волной нанотрубки. Бегущая вправо волна (фонон) нанотрубки с волновым числом $q \in[0, \pi]$, частотой $\omega_{i}(q)$ и собственным комплексным вектором $\mathbf{w}_{i}(q)$, $i=1, \ldots, 3 K$ (см. уравнение (11)) имеет вид

$\mathbf{x}_{n}(t)=\mathbf{x}_{n}^{0}+A[\operatorname{Re}(\mathbf{w}) \cos (q n-\omega t)-\operatorname{Im}(\mathbf{w}) \sin (q n-\omega t)]$,

где частота $\omega=\omega_{i}(q)$, нормированный вектор $\mathbf{w}=\mathbf{w}_{i}(q)$. Амплитуда волны $A$ определяет ее энергию $E=M A^{2} \omega^{2}$, поэтому амплитуду фонона, осуществляющего теплоперенос, можно найти из условия $M A^{2} \omega^{2}=k_{B} \Delta T / 2$, амплитуда $A=\left(k_{B} \Delta T / 2 M \omega^{2}\right)^{1 / 2}$.

Возьмем УНТ $(5,5)$ из $N=61$ звеньев, расположенную вдоль оси $x$ (координаты атомов нанотрубки $\left\{\mathbf{x}_{n}^{0}\right\}_{n=1}^{N}$ ). В центр нее поместим атом Аи (возьмем его координаты $x=(N+1) a / 2, y=0, z=0)$ и найдем $x$ компоненту силы $F_{0}$ взаимодействия частицы с атомами 


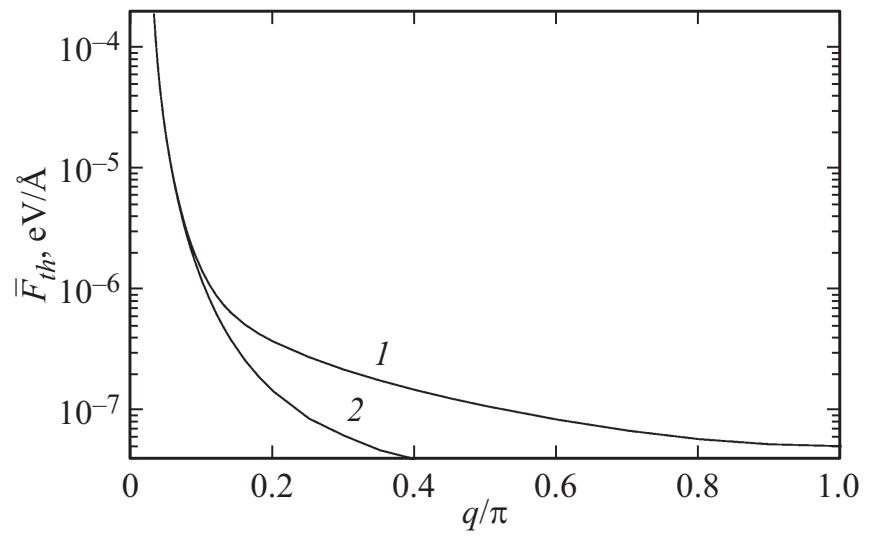

Рис. 10. Зависимость силы взаимодействия частицы $F_{t h}$ со всеми тепловыми фононами нанотрубки $(5,5)$ от волнового числа фононов $q$ при разнице температур $\Delta T=60 \mathrm{~K}$ (кривая 1). Кривая 2 дает силу взаимодействия только с изгибными фононами $F_{t}$.

нанотрубки. Далее возьмем уже нанотрубку с координатами $\left\{\mathbf{x}_{n}(t)\right\}_{n=1}^{N}$, соответствующими бегущей волне $(18)$, и снова найдем $x$ компоненту силы $F_{i}(t)$. Тогда среднее значение силы воздействия частицы с бегущей волной

$$
F_{i}(q)=\frac{1}{t_{\omega}} \int_{0}^{t_{\omega}}\left(F_{i}(\tau)-F_{0}\right) d \tau, \quad i=1,2, \ldots, 3 K,
$$

где период колебания $T_{\omega}=2 \pi / \omega$. Отсюда уже можно найти среднее значение силы воздействия на частицу всех термически активированных фононов с волновым числом $q$ :

$$
F_{t h}(q)=\sum_{i=1}^{3 K} F_{i}(q), \quad F_{t}(q)=F_{1}(q)+F_{2}(q),
$$

где вторая сумма дает вклад изгибных фононов в силу термофореза.

Зависимости сил взаимодействий $F_{t h}, F_{t}$ от волнового числа фононов $q$ представлены на рис. 10. Как видно из рисунка, основной вклад в силу термофореза дают взаимодействия с длинноволновыми изгибными фононами с волновым числом $q<0.1$. Аналогичный вывод для термофореза наночастиц на поверхности графена получен в [22].

Длинноволновые изгибные фононы нанотрубки имеют аномально большую длину пробега, поэтому сила термофореза $F_{t h}$ и скорость установившегося движения частицы $v_{s}$ слабо меняются с увеличением длины нанотрубки. Это делает термофорез в нанотрубках особо эффективным.

Продольные акустические фононы мало влияют на термофорез частиц. Для иллюстрации этого промоделируем термофорез атома $\mathrm{Au}$ в нанотрубке $(5,5)$ при учете только продольных движений атомов нанотрубки. Для этого в системе уравнений движений (15) нужно запретить смещения атомов по направлениям $y$ и $z$. Как видно из рис. 6 , в результате этого скорость движения частицы резко уменьшается. Таким образом, продольные длинноволновые акустические фононы дают значительно меньший вклад в термофорез частиц внутри нанотрубок, чем изгибные фононы.

\section{7. Заключение}

Проведенное численное моделирование показывает, что термофорез частиц (атомов) внутри одностенных углеродных нанотрубок обладает высокой эффективностью. Под действием разницы температур на концах нанотрубки $\Delta T$ в ней возникает постоянный тепловой поток $J$, вызывающий направленное движение частиц от теплого конца к холодному. Помещение частицы внутрь нанотрубки приводит к ее монотонному движению в направлении теплопереноса с постоянной скоростью $v_{s}$, величина которой слабо зависит от длины нанотрубки.

Тепловой поток вдоль нанотрубки приводит к образованию действующей на частицы постоянной силы (силы термофореза), направление которой совпадает с направлением теплопереноса. Величина силы не пропорциональна величине теплового потока $J$, а пропорциональна только разнице температур $\Delta T$. При фиксированном значении $\Delta T$ значение силы с увеличением длины нанотрубки убывает значительно медленнее величины теплового потока. Анализ взаимодействия частицы с тепловыми фононами нанотрубки (с бегущими в направлении теплопереноса волнами) показал, что величина силы термофореза практически полностью определяется взаимодействием частицы с длинноволновыми изгибными фононами, обладающими большой длиной пробега. Поэтому режим термофореза частиц внутри нанотрубок является баллистическим, а не диффузионным.

При моделировании термофореза рассматривалась только одноатомная частица, так как одноатомность позволяет напрямую вычислить силу термофореза и силу ее взаимодействия с отдельными тепловыми фононами. Полученные результаты будут конечно справедливы и для многоатомных наночастиц двигающихся внутри нанотрубок.

\section{Финансирование работы}

Научно-исследовательская работа выполнена за счет субсидии, выделенной ФИЦХФ РАН на выполнение государственного задания, тема 0082-2014-0013. Вычислительные ресурсы предоставлены межведомственным суперкомпьютерным центром РАН.

\section{Конфликт интересов}

Авторы заявляют, что у них нет конфликта интересов. 


\section{Список литературы}

[1] A. Barreiro, R. Rurali, E.R. Hernández, J. Moser, T. Pichler, L. Forró, A. Bachtold. Science 320, 775 (2008).

[2] Л.В. Радушкевич, В.М. Лукьянович. Журн. физ. химии 26, 1,88 (1952).

[3] S. Iijima. Nature 354, 56 (1991).

[4] А.В. Елецкий. УФН 172, 4, 401 (2002).

[5] P. Kim, L. Shi, A. Majumdar, P.L. McEuen. Phys. Rev. Lett. 87, 215502 (2001).

[6] P.A.E. Schoen, J.H. Walther, S. Arcidiacono, D. Poulikakos, P. Koumoutsakos. Nano Lett. 6, 9, 1910 (2006).

[7] P.A.E. Schoen, J.H. Walther, D. Poulikakos, P. Koumoutsakos. Appl. Phys. Lett. 90, 253116 (2007).

[8] J. Shiomi, S. Maruyama. Nanotechnology 20, 055708 (2009).

[9] E. Oyarzua, J.H. Walther, C.M. Megaridis, P. Koumoutsakos, H.A. Zambrano. ACS Nano 11, 10, 9997 (2017).

[10] R. Rajegowda, S.K. Kannam, R. Hartkamp, S.P. Sathian. Nanotechnology 28, 155401 (2017).

[11] E. Oyarzua, J.H. Walther, H.A. Zambrano. Phys. Chem. Chem. Phys. 20, 5, 3672 (2018).

[12] Q. Cao. J. Phys. Chem. C 123, 29750 (2019).

[13] A. Panahi, P. Sadeghi, A. Akhlaghi, M.H. Sabour. Diamond Rel. Mater. 110, 108105 (2020).

[14] H.A. Zambrano, J.H. Walther, R.L. Jaffe. J. Chem. Phys. 131, 241104 (2009)

[15] M.V.D. Prasad, B. Bhattacharya. Nano Lett. 16, 4, 2174 (2016).

[16] M.V.D. Prasad, B. Bhattacharya. Nano Lett. 17, 4, 2131 (2017).

[17] R. Rurali, E.R. Hernúndez. Chem. Phys. Lett. 497, 62 (2010).

[18] N. Wei, H.-Q. Wang, J.-C. Zheng. Nanoscale Res. Lett. 7, 1, 154 (2012).

[19] A.V. Savin, Y.S. Kivshar. Sci. Rep. 2, 1012 (2012).

[20] M. Jafary-Zadeh, C.D. Reddya, Y.-W. Zhang. Phys. Chem. Chem. Phys. 16, 5, 2129 (2014).

[21] M. Becton, X. Wang. J. Chem. Theory Comput. 10, 2, 722 (2014).

[22] E. Panizon, R. Guerra, E. Tosatti. PNAS 114, 34, E7035 (2017).

[23] A.V. Savin, Y.S. Kivshar, B. Hu. Phys. Rev. B 82, 195422 (2010).

[24] W.D. Luedtke, U. Landman. Phys. Rev. Lett. 82, 19, 3835 (1999).

[25] A.V. Savin, B. Hu, Y.S. Kivshar. Phys. Rev. B 80, 195423 (2009).

[26] А.В. Савин, О.И. Савина. ФТТ 61, 2, 409 (2019).

Редактор Ю.Э. Китаев 